\title{
A NOTE ON TWO WEIGHT FUNCTION CONDITIONS FOR A FOURIER TRANSFORM NORM INEQUALITY
}

\author{
BENJAMIN MUCKENHOUPT ${ }^{1}$
}

\begin{abstract}
Recent papers have given two different conditions on pairs of nonnegative weight functions that insure that a Fourier transform norm inequality holds in $\mathbf{R}^{n}$. With additional assumptions these conditions were also shown to be implied by the norm inequality. A direct proof is given here that these conditions are equivalent; this can be used to simplify some of the proofs in those papers.
\end{abstract}

1. Introduction. In [2] it was shown that if $1<p \leqslant q<\infty, U(x)$ and $V(x)$ are nonnegative and there is a constant $D$ such that for all $s>0$,

$$
\left[\int_{0}^{s} U^{*}(x) d x\right]^{1 / q}\left[\int_{0}^{1 / s} W(x) d x\right]^{1 / p^{\prime}} \leqslant D,
$$

where $p^{\prime}=p /(p-1)$ and $U^{*}(x)$ and $W(x)=\left[V(x)^{-1 /(p-1)}\right]^{*}$ are the nonincreasing rearrangements of $U(x)$ and $V(x)^{-1 /(p-1)}$, respectively, then for every integrable function $f$ on $\mathbf{R}^{n}$,

$$
\left[\int_{\mathbf{R}^{n}}|\hat{f}(x)|^{q} U(x) d x\right]^{1 / q} \leqslant C\left[\int_{\mathbf{R}^{n}}|f(x)|^{p} V(x) d x\right]^{1 / p},
$$

where $C$ is independent of $f$ and $\hat{f}$ denotes the Fourier transform

$$
\hat{f}(x)=\int_{-\infty}^{\infty} e^{-i x \cdot t} f(t) d t .
$$

Furthermore, it was shown that if $U(x)$ and $V(x)$ are radial, $U(x)$ is decreasing as a function of $|x|$ and $V(x)$ is increasing as a function of $|x|$, then (1.2) implies (1.1). This result with the assumption that $p$ and $q$ are not both equal to 2 was also obtained independently in [1].

With the same notation, it was shown in [3] that if $1<p \leqslant q<\infty, U(x)$ and $V(x)$ are nonnegative and there exist constants $A$ and $B$ such that for all $r>0$,

$$
\begin{array}{ll}
{\left[\int_{\left[x U^{*}(x)\right]^{p^{\prime} / q}>B r x} U^{*}(x) d x\right]^{1 / q}\left[\int_{V(x)<r^{p-1}} V(x)^{-1 /(p-1)} d x\right]^{1 / p^{\prime}} \leqslant A,} & q \leqslant p^{\prime}, \\
{\left[\int_{r U(x)>1} U(x) d x\right]^{1 / q}\left[\int_{[x W(x)]^{q / p^{\prime}}>B r x} W(x) d x\right]^{1 / p^{\prime}} \leqslant A,} & q>p^{\prime},
\end{array}
$$

Received by the editors June 15, 1982.

1980 Mathematics Subject Classification. Primary 42A38, 42B10.

'Supported in part by N.S.F. grant MCS80-03098-01. 
then for every integrable function $f$ on $\mathbf{R}^{n}$ inequality (1.2) holds with $C$ independent of $f$. With the same monotonicity conditions as in [2], (1.2) was also shown to imply (1.3).

Because of these results, it follows that (1.1) and (1.3) are equivalent conditions for $1<p \leqslant q<\infty$ since they are both conditions only on the distribution functions of $U$ and $V$. The purpose of this paper is to give a direct proof of the following more general result.

THEOREM 1. If $1<p<\infty, 1<q<\infty$ and $U(x)$ and $V(x)$ are nonnegative functions, then there is a $D$ such that (1.1) holds for all $s>0$ if and only if there exist constants $A$ and $B$ such that (1.3) holds for all $r>0$.

Besides satisfying the curiosity of people who have read these papers and wondered why (1.1) and (1.3) are equivalent, this proof also leads to simpler proofs of some of the results in [ 2 and 3 ] quoted above. In particular, the proof given here that (1.1) implies (1.3) can be combined with the proof in [3] that (1.3) implies (1.2). This gives a simpler proof that (1.1) implies (1.2) than that given in [2], especially in the case $q=p^{\prime}$. Similarly, the fact proved in [3] that (1.2) implies (1.3) can be simplified. The proof that (1.2) with the monotonicity assumptions implies (1.1) is short; this is done in [1 and 2]. It consists essentially of letting $f(x)=$ $V(x)^{-1 /(p-1)} \chi_{B}(x)$, where $B$ is the sphere with center at the origin and measure $s$ and using the fact that $|\hat{f}(x)|$ is bounded below by $\frac{1}{2} \int_{B} V(t)^{-1 /(p-1)} d t$ if $|x|$ is less than the reciprocal of the radius of $B$. This implication and Theorem 1 show that (1.2) with the monotonicity conditions implies (1.3).

Throughout this paper, the convention $0 \cdot \infty=0$ is used.

2. Proof that (1.1) implies (1.3). We will first prove this for the case $q \leqslant p^{\prime}$. To do this, fix an $r>0$ and $p$ and $q$ satisfying $1<q \leqslant p^{\prime}<\infty$. Let $B=D^{p^{\prime}}$ and $A=D$. We will prove that (1.3) holds for this $r, A$ and $B$.

To do this, let $s$ be a number such that

$$
U^{*}(s)>(B r s)^{q / p^{\prime}} / s ;
$$

if there is no such $s$, the first integral in (1.3) is 0 and (1.3) holds because of the convention $0 \cdot \infty=0$. Since $U^{*}$ is nonincreasing, $U^{*}(x) \geqslant(B r)^{q / p^{\prime}} S^{\left(q / p^{\prime}\right)-1}$ for $x$ in $[0, s]$, and from this we see that

$$
(B r s)^{q / p^{\prime}} \leqslant \int_{0}^{s} U^{*}(x) d x
$$

Since $W$ is nonincreasing on $[0,1 / s]$, we also have

$$
\frac{W(1 / s)}{s} \leqslant \int_{0}^{1 / s} W(x) d x
$$

Multiplying the $1 / q$ power of (2.2) by the $1 / p^{\prime}$ power of $(2.3)$ and using (1.1) then shows that

$$
(B r s)^{1 / p^{\prime}} s^{-1 / p^{\prime}} W(1 / s)^{1 / p^{\prime}} \leqslant D
$$


or $W(1 / s) \leqslant 1 / r$. Since $W$ is nonincreasing

$$
\int_{V(x)^{-1 /(p-1)}>1 / r} V(x)^{-1 /(p-1)} d x=\int_{W(x)>1 / r} W(x) d x \leqslant \int_{0}^{1 / s} W(x) d x .
$$

Using this in (1.1) shows that

$$
\left[\int_{0}^{s} U^{*}(x) d x\right]^{1 / q}\left[\int_{V(x)<r^{p-1}} V(x)^{-1 /(p-1)} d x\right]^{1 / p^{\prime}} \leqslant D .
$$

Since this holds for all $s$ satisfying (2.1), it also holds for

$$
s=\sup \left\{x: U^{*}(x)>(B r x)^{q / p^{\prime}} / x\right\} .
$$

With this $s$, the first integral in (2.4) is greater than or equal to the first integral in (1.3) and (1.3) follows.

The proof for $q>p^{\prime}$ follows by taking $U_{1}=V^{-1 /(p-1)}, V_{1}=U^{-1 /(q-1)}, q_{1}=p^{\prime}$, $p_{1}=q^{\prime}$ and $W_{1}=\left[V_{1}^{-1 /\left(p_{1}-1\right)}\right]^{*}=U^{*}$. Then $(1.1)$ is equivalent to

$$
\left[\int_{0}^{s} U_{1}^{*}(x) d x\right]^{1 / q_{1}}\left[\int_{0}^{1 / s} W_{1}(x) d x\right]^{1 / p^{\prime}} \leqslant D,
$$

for all $s>0$. Since $q_{1}<p_{1}^{\prime}$, the case already proved implies the existence of $A$ and $B$ such that for all $r>0$,

$$
\left[\int_{\left[x U_{1}^{*}(x)\right]^{p_{1}^{\prime} / q_{1}>B r x}} U_{1}^{*}(x) d x\right]^{1 / q_{1}}\left[\int_{V_{1}(x)<r^{p_{1}-1}} V_{1}(x)^{-1 /\left(p_{1}-1\right)} d x\right]^{1 / p_{1}^{\prime}} \leqslant A .
$$

This is equivalent to the condition in (1.3) for $q>p^{\prime}$.

3. Proof that (1.3) implies (1.1). We will prove this first for $q \leqslant p^{\prime}$. Given $s>0$, let

$$
R=\int_{0}^{1 / s} W(x) d x
$$

If $R=0$, then (1.1) follows for any $D$ from the convention $0 \cdot \infty=0$. If $0<R<\infty$, we have

$$
\int_{\{W(x) \leqslant R s / 2\} \cap[0,1 / s]} W(x) d x \leqslant \frac{R s}{2} \cdot \frac{1}{s}=\frac{R}{2},
$$

and we conclude that

$$
\int_{V(x)<[2 / R s]^{p-1}} V(x)^{-1 /(p-1)} d x=\int_{W(x)>R s / 2} W(x) d x>\frac{R}{2} .
$$

From (1.3) then

$$
\int_{\left[x U^{*}(x)\right]^{p^{\prime} / q}>B[2 / R s] x} U^{*}(x) d x \leqslant A^{q}\left[\frac{2}{R}\right]^{q / p^{\prime}} .
$$

Now

$$
\int_{\left\{\left[x U^{*}(x)\right]^{p^{\prime} / q} \leqslant B[2 / R s] x\right\} \cap[0, s]} U^{*}(x) d x \leqslant \int_{0}^{s}\left[\frac{2 B}{R s}\right]^{q / p^{\prime}} x^{\left(q / p^{\prime}\right)-1} d x .
$$


Performing the integration on the right side of (3.3) and then adding (3.2) and (3.3) gives

$$
\int_{0}^{s} U^{*}(x) d x \leqslant\left[A^{q}+\frac{p^{\prime} B^{q / p^{\prime}}}{q}\right]\left[\frac{2}{R}\right]^{q / p^{\prime}} .
$$

Now multiply the $1 / q$ power of this by the $1 / p^{\prime}$ power of (3.1) to get (1.1) with $D=2^{1 / p^{\prime}}\left[A^{q}+\left(p^{\prime} / q\right) B^{q / p^{\prime}}\right]^{1 / q}$.

If $R=\infty$, the fact that $W$ is nonincreasing shows that $\int_{0}^{t} W(x) d x=\infty$ for all $t>0$. From this it follows that

$$
\int_{V(x)<r^{p-1}} V(x)^{-1 /(p-1)} d x=\infty
$$

for all $r>0$. Then (1.3) implies that

$$
\int_{U^{*}(x)>(B r x)^{q / p^{\prime}} / x} U^{*}(x) d x=0
$$

for all $r>0$. Therefore, $U^{*}(x)=0$ for all $x>0$ and (1.1) follows for any $D$ from the convention $0 \cdot \infty=0$.

For the case $q>p^{\prime}$ use the fact that (2.5) and (2.6) are equivalent to (1.1) and (1.3), respectively, and use the case already proved to show that (2.6) implies (2.5).

\section{REFERENCES}

1. H. P. Heinig, Weighted norm inequalities for classes of operators, preprint.

2. W. B. Jurkat and G. Sampson, On rearrangement and weight inequalities for the Fourier transform, preprint.

3. B. Muckenhoupt, Weighted norm inequalities for the Fourier transform, Trans. Amer. Math. Soc. (to appear).

Department of Mathematics, Rutgers University, New Brunswick, NeW Jersey 08903 